\title{
El índice SEBI como medida del nivel de compromiso con los principios que definen la banca ética
}

\author{
SEBI Index: Measuring the commitment to the principles of social banking \\ María Eulalia Serrano Pérez
}

Universidad de Extremadura, España

Recibido el 17 de febrero de 2016; aceptado el 14 de septiembre de 2016

Disponible en Internet el 8 de julio de 2017

\begin{abstract}
Resumen
A raíz de la crisis financiera, la banca ética se ha convertido en un fenómeno visible que ha suscitado el interés tanto de clientes decepcionados de la banca convencional como de la comunidad científica. No obstante, la investigación en banca ética se encuentra aún en sus inicios, con muchos temas pendientes de consenso, como la propia definición o la identificación de los bancos considerados éticos. Teniendo en cuenta lo anterior, el propósito de este estudio es, en primer lugar, delimitar las características comunes a todos los bancos adscritos a este movimiento para, en la parte empírica, cuantificar el nivel de compromiso con el mismo y, de esta forma, como resultado de esa cuantificación, obtener un ranking que clasifique los bancos seleccionados según la puntuación obtenida. En cuanto a la metodología empleada, la cuantificación del nivel de compromiso con la banca ética se realiza a través de un nuevo índice denominado Social and Ethical Banking Index (SEBI), diseñado a partir de las características delimitadas en el estudio y aplicado a los bancos identificados como éticos por su pertenencia a la principal red de este tipo de banca: The Global Alliance for Banking on Values (GABV).

(C) 2017 Universidad Nacional Autónoma de México, Facultad de Contaduría y Administración. Este es un artículo Open Access bajo la licencia CC BY-NC-ND (http://creativecommons.org/licenses/by-nc-nd/4.0/).
\end{abstract}

Códigos JEL: G21; M14

Palabras clave: Banca ética; Banca con valores; Índice SEBI; Ranking de bancos éticos; GABV

\section{Abstract}

As a consequence of the financial crisis, social banking has become more visible and attractive for both, disappointed customers of conventional banks, and the scientific community. However, research in

Correo electrónico: mlalyserrano@gmail.com

La revisión por pares es responsabilidad de la Universidad Nacional Autónoma de México. 
social banking is still beginning. So, there are many issues without consensus, like the definition itself or the identification of social banks. Thus, this research aims to identify social banking characteristics, with the empirical objective of measuring the commitment to this movement, and ranking values-based banks according to the score obtained as a result of the quantification method that is suggested. The quantification of commitment to social banking is achieved with the new Social and Ethical Banking Index (SEBI), designed with the characteristics firstly defined. The empirical research is carried out with all the banks identified as social by their membership within the main network of social banking: The Global Alliance for Banking on Values (GABV).

(C) 2017 Universidad Nacional Autónoma de México, Facultad de Contaduría y Administración. This is an open access article under the CC BY-NC-ND license (http://creativecommons.org/licenses/by-nc-nd/4.0/).

JEL classification: G21; M14

Keywords: Social banking; Values based banking; SEBI index; Ranking of social banking; GABV

\section{Introducción}

La crisis financiera internacional ha provocado un crecimiento espectacular de la llamada banca ética, puesto que ha quedado demostrado que la industria financiera está expuesta a menudo a riesgos derivados de conductas inmorales de una magnitud tal que sobrepasa con frecuencia la del resto de sectores (Ferreira, Jalali y Ferreira, 2016). Consecuencia de ello, se observa además un interés creciente por este tema tanto de parte de los clientes de banca, como de parte de la comunidad científica.

Del lado de los clientes, estos están acudiendo masivamente a los bancos éticos, buscando una forma de hacer banca no especulativa, sino más responsable, más ética y más orientada a la comunidad que la banca tradicional. Este hecho permitió a la banca ética doblar sus activos entre 2007 y 2010, y crecer anualmente más de un $20 \%$ en el mismo periodo (Benedikter, 2011). Esta tendencia nos hace esperar en los próximos años un crecimiento anual de los bancos éticos en Europa en torno al $13-15 \%$, y tener expectativas razonables de atender a mil millones de personas en todo el mundo en 2020 (Benedikter, 2012).

Similares conclusiones se extraen de los informes de The Global Alliance for Banking on Values (GABV) «Strong, Straightforward and Sustainable Banking» y «Real Economy - Real Returns. The Power of Sustainability-focused Banking». Estos informes demuestran con datos la viabilidad de este modo de hacer banca, destacando sus ratios de rentabilidad y solvencia en comparación con los de la banca convencional (GABV, 2012, 2015). En esta línea, añadimos que otras investigaciones inciden en que los bancos éticos han demostrado un comportamiento más estable durante la crisis financiera global de 2007 (Karl, 2015).

También del lado de los clientes, tenemos que destacar la importancia de las redes sociales como factor determinante en la facilitación del desarrollo de la banca ética, debido a que, según la teoría de redes, la estructura de las relaciones sociales afecta al contexto de las relaciones que se pueden producir en la misma, por lo que es virtualmente aplicable a cualquier aspecto de la realidad social, incluida la banca (Requena, 2003).

En concreto, las redes sociales permiten incluir a la mayor parte de la población en las relaciones de confianza y simplifican las relaciones entre individuos y grupos (Fundación BBVA, 2007), potenciando de esta forma el capital social de la comunidad (los beneficios asociados a la cooperación y a la confianza entre individuos). Ello resulta decisivo a la hora de entablar relaciones de 
tipo financiero, sobre todo en países en desarrollo, si tenemos en cuenta que el capital social tiene su mayor potencial en contextos de alto riesgo y de interdependencia estratégica entre agentes (Fundación BBVA, 2007).

En el siguiente esquema del informe de la Fundación BBVA (2007) «El capital social en las comunidades autónomas y provincias» se explica gráficamente la importancia de la red social en la generalización de la confianza:

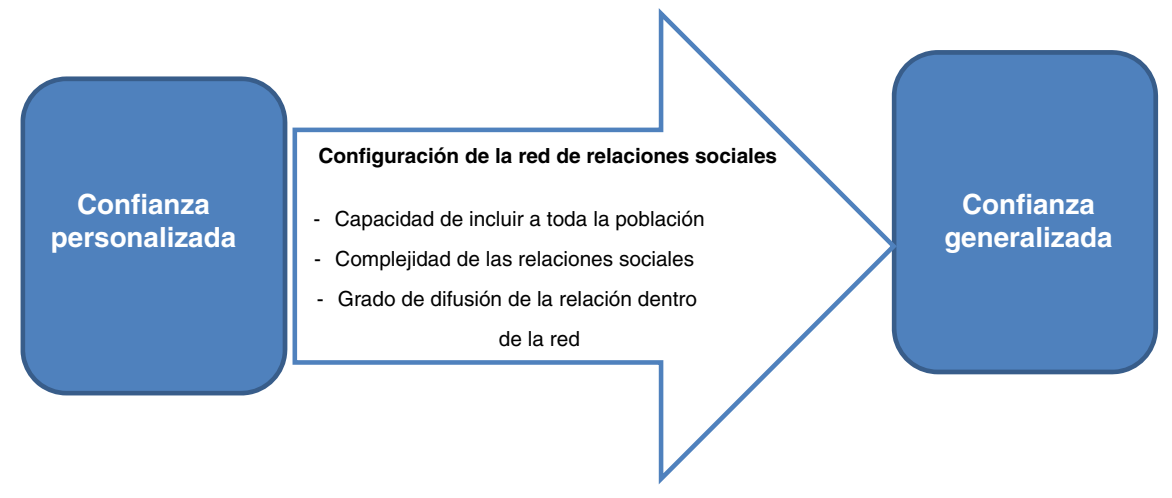

Fuente: Fundación BBVA (2007)

Continuando con el creciente interés en la banca ética por parte de la comunidad científica, podemos afirmar que la investigación sobre finanzas y banca ética se ha visto incrementada en gran medida en los últimos años. De hecho, gracias a este creciente interés han surgido nuevas instituciones, como el The Skoll Centre for Social Entrepreneurship en la Said Business School de la Oxford University, creado en 2003, o The Institute for Social Banking (ISB) en Bochum, Alemania, creado en 2006 (Benedikter, 2012).

No obstante, a pesar del interés creciente, se puede afirmar que la investigación académica en banca ética está prácticamente en sus inicios. Así lo reconocen trabajos precedentes, que afirman que la investigación en banca ética es muy insuficiente, con temas pendientes de consenso como la propia definición de banca ética, investigaciones pendientes de desarrollo sobre la identificación de las entidades que pertenecen a este movimiento, la medición de la creación de valor que aportan, la clarificación de sus objetivos, el desarrollo de una metodología común que permita medir su impacto teniendo en cuenta los distintos modelos de negocio, etc. (Bosheim, 2012).

En el mismo sentido se expresa el informe «El sistema financiero que necesitamos», realizado por el Programa de las Naciones Unidas para el Medio Ambiente (PNUMA), que reconoce que el siguiente paso crítico en la evolución hacia sistemas financieros más sostenibles es el desarrollo de un rico ecosistema de estudios y análisis que profundicen en los fundamentos teóricos y empíricos (PNUMA, 2015).

Al déficit de investigación hay que añadir la circunstancia de que la adscripción al movimiento de banca ética es completamente voluntaria, sin que además exista ningún tipo de regulación específica ni ningún registro público que identifique este tipo de bancos. Ni siquiera existen certificaciones o sellos de calidad que reconozcan el carácter social y ético de estas entidades (Sanchis, 2016).

Teniendo en cuenta lo anterior, nuestra propuesta de investigación se ha desarrollado con el objetivo de dotar a estas entidades de un mecanismo que las identifique, les sirva como herramienta de comunicación de sus principales características, les otorgue mayor credibilidad y favorezca la confianza de sus clientes, actuales y potenciales, aspectos todos ellos que deben desarrollarse al máximo para que la banca ética explote todo su potencial en el futuro (Alsina, 2002). 
En este sentido, nuestra aportación consiste en la definición de las principales características de la banca ética y la medición de la presencia de las mismas en cada uno de los bancos éticos identificados como tales a través de un nuevo índice. De esta forma, este índice medirá el nivel de compromiso de una entidad con los principios propios del movimiento de banca ética.

Para diseñar este índice se ha revisado la literatura disponible, así como los contenidos de las páginas web de los bancos éticos adheridos a la GABV.

No obstante, con carácter previo al diseño del índice, hemos investigado sobre la evolución y las características principales del movimiento de banca ética con el fin de dotar de un marco teórico adecuado a nuestra investigación empírica. Destacamos la información más relevante a nuestro juicio:

- El movimiento de banca ética intenta invertir el progresivo distanciamiento entre ética y economía que ha caracterizado la evolución de esta última (Alsina, 2002). En concreto, la banca ética defiende que el dinero no deja de ser solo un instrumento al servicio de las personas, no un fin en sí mismo (De Castro, 2013), que el beneficio debe existir, pero en su sitio, al final de la cadena (Melé, 2009), porque el valor del dinero está en las relaciones que favorece y en el bien que crea (Biggeri, 2015).

- De acuerdo con la literatura revisada, son dos las expresiones que han sido aceptadas de forma generalizada: banca ética en la bibliografía en español y social banking (banca social) en el entorno anglosajón. Según algunos autores, mientras que el adjetivo social hace referencia a la actividad del banco, el adjetivo ético la identifica más con su motivación (Alsina, 2002). Otra terminología que está comenzado a difundirse con bastante aceptación es banca sostenible, banca con valores, incluso banca regenerativa o alternativa (GABV, 2015).

- Como ya se ha expuesto, la definición de banca ética no es algo sobre lo que exista consenso (Bosheim, 2012). No obstante, por su claridad y concisión podemos destacar la que la define como la banca que pretende lograr un impacto positivo en las personas y el medio ambiente por medio de la actividad bancaria (Weber y Remer, 2011). En este sentido, es importante destacar que todas las entidades que pertenecen al movimiento de banca ética entienden el dinero no como un fin en sí mismo, sino como un instrumento para mejorar la calidad de la vida de las personas, de forma que con su uso se pueda obtener un mayor impacto en el desarrollo humano (De Clerk, 2009).

- Las características de las entidades que se consideran éticas las hemos obtenido fundamentalmente de la definición del ISB, que incluye un listado de características consensuado con todos sus miembros. A este listado hemos añadido las características extraídas de la revisión de la literatura disponible, en aras de obtener una relación de características lo más completa posible. Se expone más adelante.

\section{Metodología}

La metodología empleada para abordar la cuantificación que pretendemos es la de trabajos de investigación similares previos: metodología de construcción de índices y metodología de análisis de contenido.

Hay que insistir en que la investigación en banca ética está prácticamente en sus inicios y es del todo incompleta, por lo que aún son muy limitados los estudios empíricos realizados sobre este tema, destacando una investigación de 2003 sobre el espacio que puede haber para la banca ética desde la perspectiva del márketing (San Emeterio y Retolaza, 2003) y la realizada por San 
José y Retolaza (2007) para contrastar las diferencias entre la banca ética y el resto de entidades bancarias.

Este último trabajo, más próximo al nuestro, propone elaborar un índice nuevo, el Radical Affinity Index (RAI), como indicador cuantitativo del compromiso ético de una entidad financiera de cualquier tipo. Este índice se construye sobre cuatro indicadores sintéticos: transparencia, valor social añadido de los activos, exigencia de garantías y participación en la toma de decisiones de todos los grupos de interés (San José y Retolaza, 2007). Cada indicador incluye una serie de variables que señalan distintos niveles de compromiso ético con los que pretenden concluir las diferencias existentes entre la banca ética y la tradicional.

Cabe señalar que el RAI ha sido utilizado en los trabajos de San José, Retolaza y GutiérrezGoiria (2011), Herden (2012) y Bosheim (2012). No obstante, en nuestro caso se ha optado por construir un nuevo índice, aunque basado en la metodología del RAI, por los motivos siguientes:

- Divergencia en los objetivos. El RAI está enfocado a localizar las diferencias entre la banca ética y la banca tradicional, mientras que nuestro objetivo es cuantificar el compromiso ético y social de las entidades que pertenecen al movimiento de banca ética.

- Concepto restrictivo de banca ética. Los cuatro indicadores del RAI están basados en un marco teórico que enfatiza las variables seleccionadas para su diseño y que, en nuestra opinión, deja fuera otras variables importantes. Entendemos que, con ello, el RAI se decanta por un determinado modelo de bancos éticos, lo que, a nuestro juicio, lo invalida para nuestro objetivo de emplear una herramienta flexible que permita analizar la banca ética en toda su diversidad, teniendo en cuenta que este movimiento integra realidades y modelos de negocio muy diferentes (Sunyer y Alsina, 1999; GABV, 2015).

Dicho lo anterior, se ha optado por crear un índice nuevo, denominado Social and Ethical Banking Index (SEBI), para medir el compromiso de cada banco ético con los principios de la banca ética y, posteriormente, elaborar un ranking de bancos éticos con los resultados obtenidos.

La metodología aplicada en la cuantificación de las variables se basa en el análisis de contenido, técnica que se utiliza en la investigación científica para sistematizar la información aplicando la definición clásica de Berelson (1952): técnica de investigación para la descripción objetiva, sistemática y cuantitativa del contenido manifiesto de las comunicaciones con el fin de interpretarla (Espín, 2002).

En nuestro caso, realizaremos el análisis de contenido de los sitios web de las entidades que pertenecen a la GABV, cuyos socios constituyen la población que será objeto de nuestro análisis, considerando que la GABV es la red de bancos éticos más relevante y cuyos principios se ajustan de forma completa a los objetivos de nuestro trabajo.

Se incluye una relación de los bancos analizados en la tabla 1. En total, 27 bancos: 9 europeos, 10 de países desarrollados del resto de continentes (EE. UU., Canadá y Australia) y 8 de países en vías de desarrollo.

En la misma tabla 1 se señala la pertenencia, en su caso, al ISB, a la Federación Europea de Bancos Éticos y Alternativos (FEBEA) y/o a la International Association of Investors in the Social Economy (INAISE), entendiendo que es un dato de interés conocer la pertenencia a otras redes por dos motivos: 1) nos revela la importancia que otorga el movimiento de banca ética al trabajo en red, y 2) nos puede permitir conocer la relevancia de cada una de las redes, aunque teniendo en cuenta que GABV e INAISE son redes de ámbito internacional, mientras que ISB y FEBEA son organizaciones de ámbito europeo. 
Tabla 1

Población objeto de análisis: bancos miembros de GABV

\begin{tabular}{|c|c|c|c|c|c|}
\hline Banco & País & GABV & ISB & FEBEA & INAISE \\
\hline Affinity Credit Union (B01) & Canadá & $\mathrm{X}$ & & & \\
\hline Alternative Bank Schweiz AG (B02) & Suiza & $\mathrm{X}$ & $\mathrm{X}$ & & \\
\hline Assiniboine Credit Union (B03) & Canadá & $\mathrm{X}$ & & & \\
\hline Banca Popolare Etica (B04) & Italia & $\mathrm{X}$ & $\mathrm{X}$ & $\mathrm{X}$ & \\
\hline Banco Fie (B05) & Bolivia & $\mathrm{X}$ & & & $\mathrm{X}$ \\
\hline Banco Sol (B06) & Bolivia & $\mathrm{X}$ & & & \\
\hline Bank Australia (B07) & Australia & $\mathrm{X}$ & & & \\
\hline Beneficial State Bank (B08) & EE. UU. & $\mathrm{X}$ & & & \\
\hline BRAC (B09) & Bangladesh & $\mathrm{X}$ & & & \\
\hline Centenary Bank (B10) & Uganda & $\mathrm{X}$ & & & \\
\hline City First Bank of DC (B11) & EE. UU. & $\mathrm{X}$ & & & \\
\hline Clean Energy Development Bank (B12) & Nepal & $\mathrm{X}$ & & & \\
\hline Crédit Coopératif (B13) & Francia & $X$ & & $\mathrm{X}$ & \\
\hline Cultura Sparebank (B14) & Noruega & $\mathrm{X}$ & $\mathrm{X}$ & $\mathrm{X}$ & \\
\hline Ecology Building Society (B15) & R. Unido & $\mathrm{X}$ & $\mathrm{X}$ & & $\mathrm{X}$ \\
\hline Ekobanken (B16) & Suecia & $\mathrm{X}$ & $\mathrm{X}$ & $\mathrm{X}$ & $\mathrm{X}$ \\
\hline First Green Bank (B17) & EE. UU. & $\mathrm{X}$ & & & \\
\hline GLS Bank (B18) & Alemania & $\mathrm{X}$ & $\mathrm{X}$ & & $\mathrm{X}$ \\
\hline Merkur (B19) & Dinamarca & $X$ & $\mathrm{X}$ & $\mathrm{X}$ & $X$ \\
\hline New Resource Bank (B20) & EE. UU. & $\mathrm{X}$ & & & \\
\hline Banco & País & GABV & ISB & FEBEA & INAISE \\
\hline SAC Apoyo Integral, S.A. (B21) & El Salvador & $\mathrm{X}$ & & & \\
\hline Southern Bancorp (B22) & EE. UU. & $\mathrm{X}$ & & & \\
\hline Sunrise Banks (B23) & EE. UU. & $\mathrm{X}$ & & & \\
\hline Triodos Bank (B24) & Holanda & $X$ & $\mathrm{X}$ & & $X$ \\
\hline Vancity (B25) & Canadá & $\mathrm{X}$ & & & \\
\hline Visión Banco (B26) & Paraguay & $X$ & & & \\
\hline XacBank (B27) & Mongolia & $\mathrm{X}$ & & & \\
\hline
\end{tabular}

Fuente: elaboración propia a partir de páginas web de GABV, ISB, FEBEA e INAISE.

Cabe señalar que esta selección no es todo lo completa que desearíamos porque deja fuera los bancos éticos no pertenecientes a esta red, como es el caso del banco ético más conocido del mundo: el Grameen Bank de Bangladesh, cuyo fundador, Muhammad Yunus, cuenta con el Nobel de la Paz y el Príncipe de Asturias de la Concordia. No obstante esta limitación, a falta de bases de datos, certificaciones o regulaciones que identifiquen de forma inequívoca a los bancos éticos, hemos optado por la pertenencia a la GABV como el mejor criterio de identificación por su claridad y sencillez de aplicación.

Una vez delimitada la población objeto de estudio, pasamos a exponer el diseño del índice SEBI como medida del compromiso con los principios de la banca ética.

\section{Construcción del índice SEBI: diseño de indicadores, pre-test y delimitación de variables}

Como ya se ha señalado, el diseño del índice SEBI parte del trabajo realizado por San José y Retolaza (2007), añadiendo a las variables consideradas por estos autores el resto de características definitorias de la banca ética según la literatura revisada, principalmente las contenidas en la 
definición del ISB (2011) por su exhaustividad. Todas ellas se tendrán en cuenta en el diseño de nuestro índice.

Listamos a continuación dichas características. A efectos de facilitar la compresión del diseño del índice SEBI que se expondrá más adelante, se codifican con la letra C más un número de referencia por orden de lista.

- C1: Negative Screening. Aplican criterios negativos sociales, culturales, ecológicos y éticos para descartar proyectos con posibles impactos negativos en la sostenibilidad y el bien común. Previo al análisis financiero.

- C2: Positive Screening. Aplican criterios positivos sociales, culturales, ecológicos y éticos para apoyar proyectos con impactos positivos en la sostenibilidad y el bien común. Previo al análisis financiero.

- C3: Ofrecen servicios financieros a los excluidos del sistema financiero.

- C4: Fomentan el debate público sobre los principales retos de nuestro mundo.

- C5: Dialogan con un grupo muy amplio de stakeholders o grupos de interés.

- C6: Practican y divulgan los valores que inspiran todas sus actividades.

- C7: Consideran la sostenibilidad como valor fundamental.

- C8: Prestan especial atención a los derechos humanos y a la solidaridad.

- C9: El enfoque de su negocio es humano y humanizante.

- C10: Las formas de gobierno y sus estructuras organizacionales se basan en la participación.

- C11: Tienen estructuras jurídicas que previenen la dependencia de intereses particulares dominantes.

- C12: Defienden la igualdad de oportunidades para todos los empleados.

- C13: Ofrecen a sus clientes la posibilidad de realizar donaciones a distintas organizaciones y proyectos.

- C14: Realizan una amplia labor de concienciación.

- C15: Rechazan la maximización del beneficio como fin en sí mismo y la actividad especulativa.

- C16: Conciben el beneficio económico como medio para lograr el desarrollo social y la defensa del medio ambiente, y no como fin en sí mismo.

- C17: Triple bottom line aproach: buscan un triple beneficio: social, medioambiental y financiero.

- C18: Ofrecen transparencia máxima de toda la información disponible.

- C19: Son transparentes también en el desarrollo de su modelo de negocio.

Expuestas las características que tendremos en cuenta en el diseño de las variables que integran los indicadores sintéticos del índice SEBI, conviene aclarar qué entiende la literatura disponible por indicador sintético.

En concreto, un indicador sintético puede definirse como una función de un conjunto de variables, cada una de las cuales contribuye a cuantificar algún aspecto del concepto para el cual se quiere cuantificar su magnitud (López, Sánchez e Iglesias, 2003), quedando a elección del analista la selección de los indicadores iniciales, el modo de normalización y la ponderación de la información, como aspectos subjetivos de la definición de la medida sintética (Pérez et al., 2009).

De esta forma, se puede afirmar que no existe un procedimiento metodológico determinado como el más acertado para la construcción de un indicador sintético, sino que el procedimiento elegido dependerá del analista y del objetivo de su estudio (Pérez et al., 2009).

No obstante, sí hay que tener presente que un indicador debe estar basado en datos fiables, disponibles y fáciles de medir, que debe ser sencillo de comprender no solo por expertos, y debe centrarse en aspectos prácticos y claros (Rivas y Magadán, 2007). 
Para diseñar nuestros indicadores y el índice SEBI hemos tenido en cuenta lo anterior, priorizando la fiabilidad de los datos — disponibles todos en las páginas web de los bancos objeto de estudio- así como la sencillez de medida y de compresión a imagen del único precedente de estudio empírico similar localizado en la literatura disponible (San José et al., 2011).

En dicho estudio se evidencia el mayor peso de los indicadores transparencia y colocación de activos en proyectos de interés social, como notas diferenciadoras de la banca ética con respecto a la banca tradicional. Estos dos indicadores han sido seleccionados para el diseño de nuestro índice, añadiendo dos indicadores más con el objetivo, ya expresado, de incluir toda la diversidad de bancos éticos y todas sus características ya referidas.

De esta forma, el índice SEBI se calcula por la agregación de cuatro indicadores, cada uno de los cuales sintetiza la cuantificación de variables diseñadas considerando las características de la banca ética (C1 a C19), según se detallará posteriormente.

Se señalan a continuación los indicadores propuestos, con referencia a autores que consideran su relevancia a la hora de definir un banco como ético:

- Indicador 1 (I1). Transparencia radical, tanto de la información financiera como de toda la actividad del banco (De la Cuesta y del Río, 2001; Alsina, 2002; De la Cruz, Sasia y Garibi, 2011; Buttle, 2007; San José y Retolaza, 2007; Sasia, 2008; Bosheim, 2012; De Castro, 2013; GABV, 2015; Potts, 2014; Paulet, Parnaudeau y Relano, 2015).

- Indicador 2 (I2). Valoración ética y del impacto social, cultural y/o medioambiental de los proyectos cuya financiación se solicite, con carácter previo al obligatorio análisis financiero, como parte de la estrategia de la búsqueda del triple beneficio: social, medioambiental y financiero (Alsina, 2002; De la Cruz et al., 2011; Buttle, 2007; Sasia, 2008; San José y Retolaza, 2007; Sierra y Londoño, 2008; Melé, 2009; Scheire y Maertelaere, 2009; Goyal y Joshi, 2011; Benedikter, 2012, Bosheim, 2012; Herden, 2012; De Castro, 2013; GABV, 2015; Potts, 2014; Paulet et al., 2015; Ferreira et al., 2016).

- Indicador 3 (I3). Organizaciones con gobierno corporativo inclusivo y estructuras organizativas participativas, humanas y sostenibles que facilitan y fomentan la implicación y el compromiso con los valores de la banca ética de sus socios, de empleados y del resto de grupos de interés o stakeholders (San Emeterio y Retolaza, 2003; Sasia, 2008; Melé, 2009; Herden, 2012; Potts, 2014; GABV, 2015).

- Indicador 4 (I4). Labor de concienciación sobre la urgente necesidad de lograr un mundo más humano y sostenible (Ballesteros, 2002; Alsina, 2002; De la Cruz et al., 2006; Sasia, 2008; Sierra y Londoño, 2008; Scheire y Maertelaere, 2009; De Castro, 2013; Potts, 2014; GABV, 2015; Paulet et al., 2015).

Como hemos señalado, los indicadores anteriores se calculan cuantificando las variables o características de los bancos éticos que ya hemos listado, según la asignación que se expone en la tabla 2 y con la pretensión de incluir en nuestra cuantificación la totalidad de las características identificadas. Como aclaración previa, señalamos que el enfoque humano y humanizante del modelo de negocio (C9) es la única característica que se entiende transversal a todas las variables.

Para la delimitación de las variables incluidas en cada indicador del índice también se ha tenido en cuenta el pre-test realizado en noviembre de 2012 a la filial española de Triodos Bank, uno de los mayores bancos éticos del mundo y el mayor de Europa (Bosheim, 2012). Asimismo, se ha tenido en cuenta la información contenida en las páginas web de los bancos éticos objeto de nuestro estudio. 
Tabla 2

Asignación de características de los bancos éticos a los indicadores del modelo

\begin{tabular}{ll}
\hline Indicadores & Variables (características) \\
\hline I1: Transparencia & $\mathrm{C} 9, \mathrm{C} 18$ y C19 \\
I2: Valoración ético-social de proyectos de inversión y perspectiva triple & $\mathrm{C} 1, \mathrm{C} 2, \mathrm{C} 9, \mathrm{C} 15, \mathrm{C} 16$ y C17 \\
$\quad$ del beneficio & $\mathrm{C} 6, \mathrm{C} 7, \mathrm{C} 8, \mathrm{C} 9, \mathrm{C} 10, \mathrm{C} 11$ y C12 \\
I3: Gobierno inclusivo y estructura participativa, humana y sostenible & $\mathrm{C} 3, \mathrm{C} 4, \mathrm{C} 5, \mathrm{C} 6, \mathrm{C} 7, \mathrm{C} 8, \mathrm{C} 9, \mathrm{C} 13$ y C14 \\
I4: Labor de concienciación & \\
\hline
\end{tabular}

Fuente: elaboración propia.

Como se ha referido anteriormente, en el diseño del índice SEBI ha primado favorecer la sencillez en la medición y en la comprensión de los resultados obtenidos. Con este criterio, partimos de la hipótesis de que todos nuestros indicadores y variables tienen el mismo peso a la hora de considerar un banco como ético. Este diseño viene avalado por una gran parte de los trabajos sobre construcción de indicadores sintéticos que utilizan proyecciones lineales unidimensionales que generan medias ponderadas de indicadores simples.

De hecho, la metodología más aplicada es la que utiliza pesos de la misma cuantía y agrega la información mediante una suma, por su escasa dificultad operativa y por la facilidad asociada a la interpretación de los resultados. La ponderación y la agregación suelen hacerse en niveles sucesivos, de manera que previamente se pondera y agrega una serie de variables para construir los subindicadores relativos a una determinada dimensión para, posteriormente, obtener el indicador sintético mediante la agregación de los subindicadores (Domínguez, Blancas, Guerrero y González, 2011).

Esta metodología de agregación simple puede ser utilizada siempre que el analista requiera obtener una medida sintética computacionalmente muy sencilla y de fácil interpretación (Domínguez et al., 2011), presupuestos ambos que concurren en el diseño de nuestro índice, como ya se ha expuesto en la tabla 2.

Teniendo en cuenta lo anterior, y con la misma motivación de lograr la mayor sencillez y facilidad de interpretación y difusión, a cada indicador se le ha otorgado un 4 de valoración máxima, que se reparte de forma lineal entre sus variables, tal como se muestra en las tablas 3-6. Se obtiene de esta forma un resultado en base 16 que, para obtener el valor del índice SEBI, se

Tabla 3

Diseño indicador I1. Cuantificación de transparencia

\begin{tabular}{lc}
\hline Información publicada en sitio web & Valores \\
\hline $\begin{array}{l}\text { I1.1. Información financiera. Cuentas anuales. Información obligatoria para todas } \\
\text { las entidades financieras (Pre-test Triodos) }\end{array}$ & $1-0$ \\
I1.2. Información sobre Gobierno Corporativo y/o informes anuales sobre & $1-0$ \\
$\quad$ actividad desarrollada con información sobre impacto social, medioambiental & \\
y/o cultural de su actividad (Pre-test Triodos) & $1-0$ \\
I1.3. Información genérica sobre las operaciones en las que se coloca el activo & $1-0$ \\
$\quad$ (San José et al., 2011) & \\
I1.4. Información detallada sobre la totalidad de operaciones en las que se coloca & 4 \\
$\quad$ el activo (Pre-test Triodos; San José et al., 2011; Paulet et al., 2015) &
\end{tabular}

Fuente: elaboración propia. 
Tabla 4

Diseño indicador I2. Cuantificación de la valoración ético-social y búsqueda del triple beneficio: social, ecológico y económico

\begin{tabular}{lc}
\hline Información publicada en sitio web & Valores \\
\hline I2.1. Valoración ético-social de los proyectos o compromiso a largo plazo con los & $1-0$ \\
$\quad$ clientes y sus comunidades (Alsina, 2002; Melé, 2009; Goyal y Joshi, 2011; & \\
Benedikter, 2012; Potts, 2014; Ferreira et al., 2016; Pre-test Triodos) & $1-0$ \\
I2.2.Catálogo de criterios positivos y negativos que señalan el tipo de operaciones \\
$\quad$ financiables, o listado de sectores o grupos financiables, o detalle de tipología de \\
$\quad$ operaciones (Alsina, 2002; De la Cruz et al., 2006; Buttle, 2007; Sasia, 2008; \\
Herden, 2012; De Castro, 2013; Pre-test Triodos) \\
I2.3. Búsqueda del triple beneficio (Pre-test Triodos; Potts, 2014; Paulet et al., \\
$\quad$ 2015) \\
I2.4. Prohibición explícita de inversiones especulativas o inversión exclusiva en \\
$\quad$ proyectos que financien la economía real (Alsina, 2002; Weber y Remer, 2011; \\
$\quad$ Bosheim, 2012; Herden, 2012; De Castro, 2013; Potts, 2014; Paulet et al., 2015). \\
Total valor máximo I2
\end{tabular}

Fuente: elaboración propia.

Tabla 5

Diseño indicador I3. Cuantificación del gobierno corporativo inclusivo y estructura participativa, humana y sostenible

\begin{tabular}{lc}
\hline Información publicada en sitio web & Valores \\
\hline I3.1. No cotizan en mercados oficiales (Scheire y Maertelaere, 2009; Pre-test & $1-0$ \\
Triodos) & $1-0$ \\
I3.2. Fomentan la participación, la vinculación a la misión de la entidad y la & \\
$\quad$ cercanía a socios, empleados y todos los grupos de interés (Pre-test Triodos; San & \\
$\quad$ Emeterio y Retolaza, 2003; Sasia, 2008; Melé, 2009; Potts, 2014; Paulet et al., \\
2015) \\
I3.3 Sostenibilidad insertada en el corazón de modelo de negocio (Alsina, 2002; \\
$\quad$ Melé, 2009; Weber y Remer, 2011; Bosheim, 2012, Benedikter, 2011; Pre-test \\
$\quad$ Triodos) \\
I3.4. Se ofrecen a los empleados oportunidades de desarrollo, aplicando políticas \\
$\quad$ de igualdad para el acceso a las mismas (Sasia, 2008; Benedikter, 2012; Pre-test \\
$\quad$ Triodos) \\
Total valor máximo I3
\end{tabular}

a Ejemplos de prácticas posibles: publicación de biografías y datos de contacto de los miembros de los órganos de gobierno y principales ejecutivos, principios cooperativos, eventos participativos y periódicos, canales de comunicación abiertos a socios y stakeholders, publicación de documentos de interés para socios y stakeholders adicional al informe anual y/o memoria de gobierno corporativo (sobre eventos, legislación, novedades de interés, etc.).

Fuente: elaboración propia.

convertirá a base diez para favorecer una comprensión más intuitiva y facilitar su comprensión y difusión.

Como ya se ha expuesto, la medición de las variables se realiza a partir de la información publicada en los páginas web de las entidades analizadas, de forma que si las características que integran la variable objeto de medición están presentes se le otorga 1 punto, y si no están presentes, 0 puntos. 
Tabla 6

Diseño indicador I4. Cuantificación de la labor de concienciación

\begin{tabular}{lc}
\hline Información publicada en sitio web & Valores \\
\hline I4.1. Participación frecuente en eventos abiertos al público y/o fuerte presencia en & $1-0$ \\
las comunidades locales donde está implantada (Scheire y Maertelaere, 2009; & \\
Pre-test Triodos) & $1-0$ \\
I4.2. Publicación de blogs, boletines o revistas periódicas. En los países en & $1-0$ \\
desarrollo se valora la cercanía al cliente a través de una extensa red de & \\
colaboradores (Scheire y Maertelaere, 2009; Pre-test Triodos) & \\
I4.3. Oferta de productos que permiten donación de intereses a distintas & \\
organizaciones a clientes de bancos del primer mundo. En países en desarrollo & \\
se valora la promoción de microcréditos y la capacitación y concienciación de \\
los clientes sobre sus posibilidades de mejorar su futuro, facilitando su inclusión \\
$\quad$ en el sistema financiero (Alsina, 2002; Ballesteros, 2002; De la Cruz et al., \\
2006; Sasia, 2008; Sierra y Londoño, 2008; Scheire y Maertelaere, 2009; De \\
Castro, 2013; Potts, 2014; Pre-test Triodos) \\
I4.4. Constitución de fundaciones o fondos como receptores de donaciones, o \\
$\quad$ donaciones de la propia entidad para atender a proyectos no financiables por \\
análisis financieros negativos (Scheire y Maertelaere, 2009; Pre-test Triodos) \\
Total valor máximo I4
\end{tabular}

Fuente: elaboración propia.

La puntuación de un indicador resultará de la agregación de los valores observados para cada una de las variables que lo integran, y el valor del índice SEBI para cada banco ético se obtendrá de la agregación del valor de los cuatro indicadores en base 10:

$$
\mathrm{SEBI}=10 \cdot(\mathrm{I} 1+\mathrm{I} 2+\mathrm{I} 3+\mathrm{I} 4) / 16
$$

De acuerdo con la fórmula anterior, el valor de SEBI estará entre 0 y 10.

Cabe señalar que la razón de convertir el valor que resulta de la agregación de los indicadores a base 10 no es otra que lograr un dato lo más intuitivo posible para favorecer la comprensión y la difusión de los resultados.

\section{Resultados}

Una vez revisados los sitios web de los 27 bancos de GABV, y calculado el índice SEBI para cada uno de ellos según se ha expuesto, se han obtenido los resultados que se muestran en la tabla 7.

Del ranking anterior podemos extraer, entre otras, las siguientes conclusiones:

- De las cinco entidades que figuran en primer lugar por lograr la puntuación máxima, cuatro son europeas y una canadiense.

- Las ocho entidades que figuran en segundo lugar, con un 9.38, pierden la puntuación máxima por no informar de la totalidad de sus proyectos de inversión, a excepción de la entidad B11, que no dispone de fundación propia ni realiza donación directa de fondos, y de la B14, que no publica un catálogo de criterios positivos y negativos sobre actividades financiables. De estas siete entidades, una es de un país en desarrollo, cuatro europeas, dos norteamericanas y una canadiense. 
Tabla 7

Ranking de bancos éticos

\begin{tabular}{|c|c|c|c|c|c|c|c|c|c|}
\hline Ranking & Ref. & Banco & País & I1 & $\mathrm{I} 2$ & $\mathrm{I} 3$ & I4 & Total & SEBI \\
\hline 1 & В02 & Alternative Bank Schweiz AG & Suiza & 4 & 4 & 4 & 4 & 16 & 10 \\
\hline 1 & B04 & Banca Popolare Etica & Italia & 4 & 4 & 4 & 4 & 16 & 10 \\
\hline 1 & B18 & GLS Bank & Alemania & 4 & 4 & 4 & 4 & 16 & 10 \\
\hline 1 & B24 & Triodos Bank & Holanda & 4 & 4 & 4 & 4 & 16 & 10 \\
\hline 1 & $B 25$ & Vancity & Canadá & 4 & 4 & 4 & 4 & 16 & 10 \\
\hline 2 & B01 & Affinity Credit Union & Canadá & 3 & 4 & 4 & 4 & 15 & 9.38 \\
\hline 2 & B05 & Banco Fie & Bolivia & 3 & 4 & 4 & 4 & 15 & 9.38 \\
\hline 2 & B08 & Beneficial State Bank & EE. UU. & 3 & 4 & 4 & 4 & 15 & 9.38 \\
\hline 2 & $B 11$ & City First Bank of DC & EE. UU. & 4 & 4 & 4 & 3 & 15 & 9.38 \\
\hline 2 & B13 & Crédit Coopératif & Francia & 3 & 4 & 4 & 4 & 15 & 9.38 \\
\hline 2 & B14 & Cultura Sparebank & Noruega & 4 & 3 & 4 & 4 & 15 & 9.38 \\
\hline 2 & B15 & Ecology Building Society & R. Unido & 3 & 4 & 4 & 4 & 15 & 9.38 \\
\hline 2 & B19 & Merkur & Dinamarca & 4 & 3 & 4 & 4 & 15 & 9.38 \\
\hline 3 & B07 & Bank Australia & Australia & 3 & 4 & 4 & 3 & 14 & 8.75 \\
\hline 3 & B16 & Ekobanken & Suecia & 4 & 4 & 4 & 2 & 14 & 8.75 \\
\hline 3 & $B 20$ & New Resource Bank & EE. UU. & 3 & 4 & 3 & 4 & 14 & 8.75 \\
\hline 3 & B21 & SAC Apoyo Integral, S.A. & El Salvador & 3 & 3 & 4 & 4 & 14 & 8.75 \\
\hline 3 & $B 22$ & Southern Bancorp & EE. UU. & 3 & 4 & 3 & 4 & 14 & 8.75 \\
\hline 3 & B26 & Visión Banco & Paraguay & 3 & 3 & 4 & 4 & 14 & 8.75 \\
\hline 3 & $\mathrm{~B} 27$ & XacBank & Mongolia & 3 & 3 & 4 & 4 & 14 & 8.75 \\
\hline 4 & B03 & Assiniboine Credit Union & Canadá & 3 & 3 & 4 & 3 & 13 & 8.13 \\
\hline 4 & B06 & Banco Sol & Bolivia & 2 & 3 & 4 & 4 & 13 & 8.13 \\
\hline 4 & B09 & BRAC & Bangladesh & 3 & 3 & 3 & 4 & 13 & 8.13 \\
\hline 4 & B10 & Centenary Bank & Uganda & 2 & 3 & 4 & 4 & 13 & 8.13 \\
\hline 5 & $B 23$ & Sunrise Community Banks & EE. UU. & 1 & 3 & 4 & 4 & 12 & 7.50 \\
\hline 6 & $B 17$ & First Green Bank & EE. UU. & 1 & 2 & 4 & 3 & 10 & 6.25 \\
\hline 7 & B12 & Clean Energy Development B. & Nepal & 2 & 1 & 2 & 2 & 7 & 4.38 \\
\hline
\end{tabular}

En negrita, bancos europeos; en cursiva, bancos de países desarrollados no europeos; en redonda, bancos de países en vías de desarrollo.

Fuente: elaboración propia.

- Ningún banco ético de los que operan en países en vías de desarrollo puntúa por la publicación de la totalidad de sus proyectos de inversión, por lo que en futuras investigaciones se podría analizar si esta característica tiene sentido en bancos dedicados principalmente a conceder microcréditos con el fin de hacer posible la inclusión financiera de sus clientes objetivo.

- En la tercera posición del ranking, con un 8.75, figuran siete entidades, una europea, dos norteamericanas, una australiana y tres de países en vías de desarrollo. En la cuarta posición, con un 8.13, hay una entidad canadiense y tres de países en vías de desarrollo. En quinta posición, con un 7.50, figura un banco norteamericano.

- Los bancos que aparecen en los últimos puestos, B17 y B12, con una puntuación de 6.25 y 4.38, respectivamente, radican en EE. UU. y Nepal, también respectivamente. Estas entidades tienen puntuaciones limitadas en transparencia, no publican información sobre valoraciones ético-sociales previas de los proyectos solicitantes de financiación, y el B12 también obtiene una puntuación limitada en la información que ofrece sobre su labor de concienciación.

- En relación con los ocho bancos éticos de países en desarrollo analizados, podemos concluir que todos, salvo el B12, figuran entre las cuatro primeras posiciones. Y que en estos cuatro 
primeros puestos del ranking se concentran el $89 \%$ de los 27 bancos analizados, que obtienen una puntuación entre el sobresaliente (10) y el notable alto (8.13).

A la vista de estos resultados presentamos a continuación las principales conclusiones e implicaciones de esta investigación.

\section{Conclusiones}

Tras analizar el resultado de nuestra investigación en las páginas precedentes, se exponen a continuación las principales conclusiones que, a nuestro juicio, se derivan de la misma:

1. Los bancos éticos, en su diversidad, tienen características comunes a todos ellos. Uno de nuestros objetivos ha sido la delimitación de las características comunes a todos los bancos éticos, alcanzado gracias a la revisión de la literatura y también a la información facilitada por las principales redes de bancos éticos: ISB, GABV, FEBEA e INAISE. En concreto, hemos concluido que existen 19 características comunes, que pueden resumirse en cuatro categorías más generales:

a) Transparencia radical.

b) Búsqueda de un triple beneficio (social, medioambiental y económico), lo que incluye la valoración social y medioambiental de los proyectos, previa al análisis financiero.

c) Gobiernos corporativos inclusivos y estructuras organizativas participativas, humanas y sostenibles.

d) Labor de concienciación para extender la formación financiera, el consumo financiero responsable y la necesidad de implicarnos en un desarrollo humano y sostenible, tanto en lo local como en lo global.

2. El objetivo empírico de este trabajo ha sido la medida del compromiso de los bancos denominados como éticos con el movimiento de banca ética, a través del índice SEBI. Como premisa importante, se ha tenido en cuenta que debe ser un índice flexible que permita cuantificar toda la diversidad del espectro de bancos éticos, y que en su diseño debe primar la sencillez en el cálculo y la interpretación.

Teniendo en cuenta que la adscripción al movimiento de banca ética es totalmente voluntaria, sin que exista hoy por hoy regulación alguna sobre la misma, ni certificación pública ni privada, hemos concluido que la cuantificación es muy relevante, en tanto en cuanto puede dotar de mayor credibilidad a este fenómeno y reforzar la confianza de sus clientes actuales y potenciales.

3. Mediante el cálculo del índice SEBI para los bancos éticos de GABV se ha obtenido un ranking de bancos éticos. El ranking revela que la banca ética europea es la que presenta el mayor nivel de compromiso en general, aunque también se ha demostrado el elevado compromiso de los bancos de países en vías de desarrollo (excepto en un caso), y en general, el elevado compromiso de todos los bancos de GABV, con el $89 \%$ de ellos en los cuatro primeros puestos del ranking, con puntuaciones mínimas de 8.13.

A nuestro juicio, hay que subrayar que las anteriores conclusiones reflejan las principales aportaciones de esta investigación en relación con trabajos anteriores que han abordado otros aspectos de la cuestión, como el desarrollo de un índice que compare la banca ética con la tradicional. Asimismo, entendemos que la aportación de un ranking de bancos éticos a partir de una definición amplia, que no excluya ninguna de las características de la banca ética contempladas 
por la literatura, supone igualmente una aportación que puede abrir nuevas e interesantes líneas de investigación para el futuro.

En cualquier caso, consideramos que la posibilidad de realizar las aportaciones referidas se ha visto facilitada por lo reciente del fenómeno, que hace que la literatura científica sobre el mismo sea aún muy limitada, con muchos temas pendientes de consensuar, como la propia definición.

De la misma forma, la juventud de este movimiento ha añadido dificultades a la investigación, como el hecho de que no haya base de datos, regulaciones o certificaciones disponibles que permitan la identificación de los bancos éticos o criterios mínimos de homogeneización, puesto que la diversidad que se observa en este tipo de bancos es una dificultad en sí misma que nos ha obligado a realizar un esfuerzo de adaptación de nuestro índice a toda la casuística observada. No obstante, entendemos que este trabajo de homogeneización también puede suponer una aportación adicional, sobre la que se podrá avanzar en el futuro.

En este sentido, comentamos a continuación las futuras líneas de investigación que, a nuestro juicio, pueden resultar más interesantes:

a) Análisis de los factores determinantes de la opción por el compromiso voluntario con la banca ética de los bancos adscritos al fenómeno, partiendo de motivaciones posibles como la crisis, el tamaño, el compromiso social o el compromiso medioambiental.

b) Desarrollos posteriores del índice SEBI:

- Análisis en profundidad de las posibles manifestaciones de las distintas variables en los bancos éticos que operan en países en desarrollo, atendiendo a la especial tipología de sus clientes.

- Validación de la hipótesis utilizada en el presente trabajo sobre el peso idéntico de variables e indicadores, o propuesta de hipótesis alternativas con análisis de la ponderación que se debe otorgar a cada uno de los indicadores, y de la propuesta de cálculo de cada indicador a partir de las variables que lo integran.

c) Desarrollo de procedimientos que permitan comprobar y certificar la adscripción de una entidad al movimiento de banca ética mediante la revisión de todos los aspectos que se consideren relevantes: información, operativa, procedimientos, gestión de recursos humanos, gobierno corporativo, relaciones con el resto de grupos de interés, labor de concienciación, etc.

d) Ante el hecho de que un banco ético tan conocido y relevante como el Grameen Bank no pertenezca a ninguna red de bancos éticos, planteamos como tema añadido el análisis de los costes y beneficios que supone la pertenencia de un banco ético a las distintas redes que los integran.

\section{Referencias}

Alsina, O. (2002). La banca ética. Mucho más que dinero. Barcelona: Icaria.

Ballesteros, C. (2002). Experiencias de banca ética en Europa. Ponencia I Jornadas de reflexión sobre banca ética, organizadas por Cáritas Española y celebradas en El Escorial.

Benedikter, R. (2011). European answers to the financial crisis: Social banking and social finance [consultado 23 Ene 2013]. Disponible en: http://spice.fsi.stanford.edu/docs/european_answers_to_the_financial_ crisis_social_banking_and_social_finance

Benedikter, R. (2012). Social banking and social finance: Building stones towards a sustainable post-crisis financial system? [consultado 23 Ene 2013]. Disponible en: http://www.europeanfinancialreview.com/?p=2027

Berelson, B. (1952). Content analysis in communication research. Illinois: Free Press [citado por Espín, (2002)].

Biggeri, U. (2015). El valor del dinero. Banca, finanzas y ética más allá del mito del crecimiento. Bilbao: Sal Terrae. 
Bosheim, S.A. (2012). Social banks and impact measurement: The case of Charity Bank and Triodos Bank. ISB Paper Series, No. 10. The Institute for Social Banking [consultado 10 Ene 2013]. Disponible en: http://www.social-banking.org/fileadmin/isb/Artikel_und_Studien/Paper_Series/Paper_No.10.pdf

Buttle, M. (2007). "I'm not in for the money": Constructing and mediating ethical reconnections in U.K. social banking. Geoforum, 38, 1076-1088, http://doi.org/10.1016/j.geoforum.2006.12.011.

De Castro, M. (2013). Banca ética: ¿es posible? Dossier n. ${ }^{\circ}$ 8, 4-9. Economistas sin Fronteras [consultado 14 May 2013 ]. Disponible en: http://ecosfron.org/ecosfron/wp-content/uploads/DOSSIERES-EsF-8-BANCA-\%C3\%89TICA.pdf

De Clerk, F. (2009). Ethical banking [consultado 15 Ene 2013]. Disponible en: http://www.social-banking.org/ fileadmin/isb/Artikel_und_Studien/de_Clerck_Ethical_Banking.pdf

De la Cruz, C., Sasia, P. y Garibi, J. (2011). Lo que debe ser y es una banca ética. Lan Harremanak: Revista de Relaciones Laborales, 14, 175-204. Disponible en: http://www.ehu.eus/ojs/index.php/Lan_Harremanak/article/ viewFile/3448/3086.

De la Cuesta, M. y del Río, N. (2001). Dinero más ético y solidario para una sociedad más humana y responsable. Noticias de Economía Pública, Social y Cooperativa, 33, 46-52.

Domínguez, M., Blancas, F. J., Guerrero, F. M. y González, M. (2011). Una revisión crítica para la construcción de indicadores sintéticos. Revista de Métodos Cuantitativos para la Economía y la Empresa, 11, 41-70. Disponible en: http://www.upo.es/RevMetCuant/art.php?id=48.

Espín, J. (2002). El análisis de contenido: una técnica para explorar y sistematizar información. Revista de Educación, 4, 95-105. Disponible en: http://uhu.es/publicaciones/ojs/index.php/xxi/article/viewFile/611/934.

Ferreira, F. A. F., Jalali, M. S. y Ferreira, J. J. M. (2016). Experience-focused thinking and cognitive mapping in ethical banking practices: From practical intuition to theory. Journal of Business Research, 69(11), 4953-4958, http://doi.org/10.1016/j.jbusres.2016.04.058.

Fundación BBVA (2007). El capital social en comunidades autónomas y provincias. Tema 4 Capital y Crecimiento [consultado 4 Jul 2016]. Disponible en: http://www.fbbva.es/TLFU/dat/4_cuaderno_divulgacion_web.pdf

Global Alliance for Banking on Values (2012). Strong, Straightforward and Sustainable Banking [consultado 6 May 2013]. Disponible en: http://www.gabv.org//wp-content/uploads/Full-Report-GABV-v9d.pdf

Global Alliance for Banking on Values (2015). Real Economy - Real Returns. The Power of Sustainabilityfocused Banking [consultado 12 Ene 2016]. Disponible en: http://www.gabv.org//wp-content/uploads/GABVResearch-Real-Economies-Real-Returns-2015.pdf

Goyal, K. y Joshi, V. (2011). A study of social and ethical issues in banking industry. International Journal of Economics and Research, 2(5), 49-57. Disponible en: http://ijeronline.com/documents/volumes/Vol\%202\% 20issue\%205/ijer20110205SO(5).pdf.

Herden, L. (2012). ¿Cómo medir el impacto social de la banca ética? Un análisis de métodos posibles. Estudio del caso FIARE. Madrid: TFM. Universidad Pontificia Comillas.

Karl, M. (2015). Are Ethical and Social Bank Less Risky? Evidence from a New Dataset. DIW Berlin Discussion Paper $\mathrm{N}^{\circ} 184$ [consultado 13 Oct 2016]. Disponible en: https://www.diw.de/documents/publikationen/ 73/diw_01.c.508003.de/dp1484.pdf.

López, M. E., Sánchez, P. y Iglesias, C. L. (2003). Bienestar socioeconómico de los municipios gallegos. Revista Galega de Economía, 12, 1-24 [citado por Pérez et al. (2009)].

Melé, J. (2009). Dinero y conciencia. ¿A quién sirve mi dinero? Barcelona: Plataforma Editorial.

Paulet, E., Parnaudeau, M. y Relano, F. (2015). Banking with Ethics: Strategic Moves and Structural Changes of the Banking Industry in the Aftermath of the Subprime Mortgage Crisis. Journal of Business Ethics, 131(1), 199-207, http://doi.org/10.1007/s10551-014-2274-9.

Pérez, V. E., Blancas, F. J., González, M., Guerrero, F. M., Lozano, M., Pérez, F. y Caballero, R. E. (2009). Evaluación de la sostenibilidad del turismo rural mediante indicadores sintéticos. Revista de Investigación Operacional, 30(1), $40-51$.

Programa de las Naciones Unidas para el Medio Ambiente (2015). El Sistema Financiero que Necesitamos [consultado 17 Oct 2016]. Disponible en: http://unepinquiry.org/wp-content/uploads/2015/10/The_Financial_ System_We_Need_ES.pdf

Potts, R. (2014). Banking for the Common Good: How social banks support the re-emergence of a healthy Commons. ISB Paper Series, No. 13. The Institute for Social Banking [consultado 7 Nov 2016]. Disponible en: http://www.socialbanking.org/fileadmin/isb/Artikel_und_Studien/Paper_Series/ISB_paper_no_13__Banking_on_the_Commons.pdf.

Requena, F. (2003). Análisis de las redes sociales. Orígenes, teorías y aplicaciones (1. a ed.). España: Siglo XXI España.

Rivas, J. y Magadán, M. (2007). Los indicadores de sostenibilidad en el turismo. Revista de Economía, Sociedad, Turismo y Medio Ambiente, 6, 27-61. Disponible en: http://www.tuciudadcolonial.do/aplicacion/attachments/article/80/ Indicadores_de_sostenibilidad_en_el_turismo.pdf. 
San Emeterio, J. y Retolaza, J. L. (2003). ¿Existe espacio para una banca ética? Lan Harremanak: Revista de Relaciones Laborales, 9, 127-163. Disponible en: http://www.ehu.eus/ojs/index.php/Lan_Harremanak/article/view/5255.

San José, L. y Retolaza, J.L. (2007). Análisis comparativo de la banca ética con la banca tradicional: identificador de indicadores. Conocimiento, innovación y emprendedores: camino al futuro, 1104-1118. Catálogo coordinado por Juan Carlos Ayala Calvo. Universidad de La Rioja.

San José, L., Retolaza, J. L. y Gutiérrez-Goiria, J. (2011). Are ethical banks different? A comparative analysis using the Radical Affinity Index. Journal of Business Ethics, 100(1), 151-173, http://doi.org/10.1007/s10551-011-0774-4.

Sanchis, J. R. (2016). ¿Es posible un mundo sin bancos? Vilassart de Dalt, Barcelona: El Viejo Topo.

Sasia, P. (2008). Entender hoy la banca ética. Revista Internacional de Estudios Vascos, 53, 507-532.

Scheire, Ch. y Maertelaere, S. (2009). Banking to make a difference [consultado 12 Feb 2013]. Disponible en: http://www.social-banking.org/uploads/media/Scheire_Maertelaere_Banking_to_Make_a_Difference_01.pdf

Sierra, J. H. y Londoño, D. A. (2008). Potencial de las finanzas éticas en la generación de nuevas alternativas de inversión en Colombia. Cuadernos de Administración, 21(36), 183-220. Disponible en: http://www.redalyc.org/articulo. oa?id=20503609.

Sunyer, R. y Alsina, O. (1999). La banca ètica: una experiència consolidada. Diàlegs. Revista d'Estudis Polítics i Socials, 2(4), 143-162.

Weber, O. y Remer, S. (2011). Social Banks and de Future of Sustainable Finance. New York, NY: Routledge. 